\title{
Mediterranean Diet and Minimizing Neurodegeneration
}

\author{
Francesco Sofi • Claudio Macchi • Alessandro Casini
}

Published online: 20 March 2013

(C) Springer Science+Business Media New York 2013

\begin{abstract}
Neurodegenerative diseases, a complex of diseases that ranges from cognitive decline, dementia, to Alzheimer's disease represent, to date, a public health priority all over the world. Recently, an increasing interest for a possible relationship between diet and cognitive health has been reported. Several models of diet have been proposed but, until now, the highest attention of researchers, clinicians, and institutions has been focused on the Mediterranean diet. Mediterranean diet is a model of diet characterized by a high consumption of complex carbohydrates, fruit and vegetables, legumes, with olive oil as the principal source of fats. Mediterranean diet has been extensively reported to be associated with a favorable health outcome and a better quality of life, but inconsistencies on the possible association with neurodegenerative diseases have been reported. The purpose of this review was to examine the most updated evidence of the beneficial effects of Mediterranean diet on the occurrence of neurodegenerative diseases.
\end{abstract}

Keywords Mediterranean diet $\cdot$ Neurodegenerative diseases $\cdot$ Dementia $\cdot$ Alzheimer's disease

\section{Introduction}

Neurodegenerative diseases are conditions that are characterized by a progressive neuronal dysfunction, often

F. Sofi $(\bowtie) \cdot$ C. Macchi $\cdot$ A. Casini

Department of Clinical and Experimental Medicine,

University of Florence, Largo Brambilla 3,

50134 Florence, Italy

e-mail: francescosofi@gmail.com

F. Sofi $\cdot$ A. Casini

Agency of Nutrition, Azienda Ospedaliero-Universitaria Careggi,

Florence, Italy

F. Sofi $\cdot$ C. Macchi

Don Carlo Gnocchi Foundation Italy, Onlus IRCCS, Florence, Italy associated with atrophy of the affected central or peripheral structures of the nervous system [1]. The prevalence of neurodegenerative diseases increases exponentially with age from approximately $1 \%$ in the age group of 65-70 years to $30 \%$ at age 90 years and older. Given the ever-increasing life expectancy in the industrialized countries, the number of subjects afflicted with neurodegenerative diseases increases every year. It is estimated that 4.6 million new cases of dementia arise every year [1].

Actually, neurodegenerative diseases are not curable and current treatments have limited effectiveness [2]. Hence, increasing interest for effective preventive measures has been recently demonstrated [2,3]. A delay or prevention of the onset of such diseases by 1 year could translate into 1 million fewer numbers of cases than predicted by the year 2050. In this scenario, one of the most intriguing and appealing hypotheses is the possible association between lifestyle habits, such as diet and dietary habits, and the occurrence of dementia [4]. Modifications of lifestyle habits have been demonstrated to be highly effective in reducing the risk burden of many important chronic diseases, with a wide consensus manifested on the role of diet in the etiology of cardiovascular and chronic metabolic diseases [5]. With regard to neurodegenerative diseases, the evidence for a possible role of diet on the occurrence of dementia and Alzheimer's disease has been recently suggested $[6,7]$.

Concomitantly, an increasing interest in the prevention of so-called "mild cognitive impairment" also has been reported [8]. Mild cognitive impairment is a relevant health disorder, because it refers to the grey zone between the cognitive changes of normal aging and very early dementia. Individuals with mild cognitive impairment show a decline in cognitive function greater than that expected for their age, and this condition has been reported to be an independent risk factor for neurodegenerative diseases, such as Alzheimer's disease. In fact, subjects with mild cognitive impairment represent a high-risk group because they develop dementia at 2-7\% per year compared with the general population [9]. During the past few years, some studies have 
suggested a possible influence of several models of diet for the prevention of the progressive loss of cognitive function, which may lead to dementia and other neurodegenerative diseases, such as Alzheimer and Parkinson's diseases, but the Mediterranean diet model is probably the most interesting $[10 \bullet, 11]$.

\section{Mediterranean Diet: Historical Background}

The definition of Mediterranean diet dates back to the early 1960s when Prof. Ancel Keys described, according to the observations made during his journeys in southern Europe and, more importantly, in consideration of the results coming from the Seven Countries' Study, striking low rates of death for coronary heart disease and cancer in Mediterranean countries with respect to the other study populations. Indeed, among the 12,673 subjects aged 40 to 59 years analyzed within the seven countries (United States, Finland, the Netherlands, Italy, Greece, the former Yugoslavia, and Japan) of this pioneering study, an approximate tenfold reduction of mortality rate for cardiovascular and neoplastic diseases was observed in Mediterranean countries (Italy and Greece) with respect to the other studied populations [12]. Henceforward, the Mediterranean diet began to receive increasing attention from both scientific and lay communities and many studies have been conducted to investigate whether this type of diet could be associated with a reduced risk of mortality and morbidity in the general population. As a result, Mediterranean diet has been promoted as a model for healthy eating and it has been widely recognised to have favorable effects on lipid profile and to provide a significant source of antioxidants and vitamins [5].

\section{Mediterranean Diet: What is it and How to Measure it}

The Mediterranean-style diet is not a specific diet, but rather a collection of eating habits traditionally followed by people in the different countries bordering the Mediterranean Sea. According to the original definition of Keys, "The dietary pattern of people living in Southern Italy in the 1960s," the Mediterranean diet can be described as a dietary pattern characterized by high consumption of olive oil, vegetables, legumes, whole grain products, fruits, and nuts. The intake of saturated animal fats is relatively low and moderate fish consumption (depending on the proximity to the sea) gives enough amounts of polyunsaturated fatty acids. Finally, moderate red wine consumption during meals is the main source of alcohol intake. Nevertheless, there is not a single Mediterranean diet; dietary habits of countries bordering the Mediterranean Sea vary considerably, and relevant differences in dietary pattern exist even inside the same country.
Furthermore, the Mediterranean diet should not be considered just a dietary pattern, but an integrated way of living where foods represent only one of the relevant components along with culture, beliefs, tradition, and pleasure. This complexity explains why a general definition of such dietary pattern is difficult to obtain, as well as the methods for its evaluation. For decades, studies have been targeted to the evaluation of single dietary components of the Mediterranean diet in relation to different health outcomes. A great amount of literature supporting the evidence that an increased consumption of some food groups, such as fruits and vegetables, fish, or whole-grain cereals, is somewhat beneficial for health has been proposed. Such studies, however, adopted the translational concept that, if a dietary pattern is plenty of a specific food group, and this food group has been shown to be associated with a reduced incidence of diseases, the resulting diet will be beneficial for health. On the other hand, it seems clear that such a reductionist approach presents several conceptual and methodological limitations because food components of diet present synergistic and antagonist interactions and, more importantly, because people eat a complex of nutrients and not just isolated foods. Moreover, the failure of several recent clinical trials with single-nutrient supplementation has suggested that the global nutrition pattern, rather than specific nutrients, might have protective effects on the occurrence of disease.

Thus, research efforts in this field switched progressively to the evaluation of a score for the adherence to the Mediterranean dietary pattern, rather than to the identification of single nutrients in association with the disease. Some attempts for estimating the adherence to the whole diet, and particularly to the Mediterranean diet, have been conducted [13-16]. The first and most widely used tool to assess adherence to Mediterranean diet, created by Trichopoulou et al., has been extremely used for epidemiological research, and it is based on the gender-based median amount of consumption of food groups characteristics of the traditional Mediterranean diet in the sample investigated [13]. Another model is that of Panagiotakos et al. who created a simple questionnaire based on recommendations of the guidelines for a healthy diet and characterized by few questions used for estimating the consumption frequency of food groups that are characteristic of the Mediterranean diet [14]. A further example is the questionnaire developed in the PREDIMED study and recently assessed in the SUN cohort in Spain [15]. This questionnaire is composed of questions related to the dietary habits of the population with regard to the typical Mediterranean dietary habits. Finally, another proposed tool for measuring the adherence to Mediterranean diet is the Mediterranean Adequacy Index, a ratio between the consumption of typical Mediterranean foods and those not typical of the traditional Mediterranean diet [16]. 


\section{Mediterranean Diet and Neurodegenerative Diseases}

In recent years, the association between the Mediterranean diet measured through all the aforementioned methods and neurodegenerative diseases has been investigated [10•]. The possible influence of such diet on the prevention of these clinical disorders has been tested in cohort studies, with results converging toward the beneficial effect of a greater adherence to the Mediterranean diet versus the occurrence of dementia and Alzheimer's disease. On the other hand, some very recent studies have reported a nonsignificant association between Mediterranean dietary profile and cognitive function, thus underlying the need for further and larger studies to validate these preliminary data.

All of the studies that explored the possible association between adherence to Mediterranean diet and neurodegenerative diseases in their various clinical conditions are reported in Table 1. It is interesting that all of the studies used the adherence score proposed by Trichopoulou et al., although with some variations, and most of the published studies come from the same cohort population. Moreover, most of the studies have been conducted in extra-Mediterranean populations.

The first clinical study that opened the way of research into this interesting aspect was the prospective cohort study conducted by Scarmeas et al. in 2006 within the WHICAP project (Washington Heights-Inwood Columbia Aging Project) [17]. The authors studied a population of 2,258 subjects in whom cognitive function was evaluated every 1.5 years for a total of 4 years of follow-up. At the end of the observation period 262 cases of Alzheimer's disease were observed. A greater adherence to the Mediterranean diet was able to reduce the risk of developing Alzheimer's disease by approximately $10 \%$ (hazard ratio (HR) 0.91 ; $95 \%$ confidence interval (CI) 0.83-0.98) after adjustment for potential confounders. Moreover, subjects in the middle tertile of score had a $21 \%$ lower risk of Alzheimer's disease and those in the highest tertile had a $40 \%$ lower risk of Alzheimer's disease (HR 0.6; 95 \% CI 0.42-0.87) compared with individuals in the lowest tertile of adherence.

After this first interesting report, some other studies from the same authors and the same cohort have been published. In the same year, the authors published a nested case-control study that analyzed a group of 194 patients with Alzheimer's disease compared with a group of 1,790 nondemented subjects [18]. The purpose of this study was to investigate whether traditional cardiovascular risk factors could be implicated in the association between Mediterranean diet and Alzheimer's disease. The main result of this case-control study was that a higher adherence to Mediterranean diet was associated with a lower risk for Alzheimer's disease (odds ratio (OR) 0.76; $95 \%$ CI 0.67-0.87), thus confirming in part the results of their previous study and showing the evidence that the introduction of the vascular variables in the model did not change the magnitude of the association. In addition, subjects in the highest tertile reported a significant protection versus the occurrence of Alzheimer's disease (OR 0.32; $95 \%$ CI 0.17-0.59) compared with subjects in the lowest tertile of adherence, after multiple adjustment for several variables associated with vascular disease.

Interestingly, the same authors investigated also the relation between adherence to Mediterranean diet and mortality

Table 1 Studies investigating the association between Mediterranean diet and neurodegenerative diseases

\begin{tabular}{|c|c|c|c|c|c|}
\hline Study (Cohort) & Country & Type of study & Subjects, $\mathrm{n}$ & Outcome (n) & Association \\
\hline Scarmeas et al., [17] (WHICAP) & U.S. & Prospective (follow-up 4 years) & 2,258 & $\mathrm{AD}(262)$ & Yes \\
\hline Scarmeas et al., [18] (WHICAP) & U.S. & Case-control & $\begin{array}{l}194 \text { cases } \\
1,790 \text { controls }\end{array}$ & AD (194) & Yes \\
\hline Scarmeas et al., [19] (WHICAP) & U.S. & Prospective (follow-up 4.4 years) & 192 & Mortality in AD patients (85) & Yes \\
\hline Gao et al., [27] (HPF and NHS) & U.S. & Prospective (follow-up 16 years) & 131,368 & Parkinson disease (508) & Yes \\
\hline Scarmeas et al., [20] (WHICAP) & U.S. & Prospective (follow-up 4.5 years) & 1,393 & MCI (275) & Yes \\
\hline Feart et al., [22] (Three-City) & France & Prospective (follow-up 4.1 years) & 1,410 & $\begin{array}{l}\text { Cognitive performance } \\
\text { and dementia (99) }\end{array}$ & $\begin{array}{l}\text { Yes, cognitive } \\
\text { performance } \\
\text { No, dementia }\end{array}$ \\
\hline Scarmeas et al., [21] (WHICAP) & U.S. & Prospective (follow-up 5.4 years) & 1,880 & $\mathrm{AD}(282)$ & Yes \\
\hline Roberts et al., [23] & U.S. & Prospective (follow-up 2.2 years) & 1,233 & MCI (93) Dementia (23) & No \\
\hline Tangney et al., [26] (CHAP) & U.S. & Prospective (follow-up 7.6 years) & 3,790 & Decline of cognitive function & Yes \\
\hline Cherbuin \& Anstey, [24] & Australia & Prospective (follow-up 4 years) & 1,528 & MCI (37) & No \\
\hline $\begin{array}{l}\text { Kesse-Guyot et al., [25••] } \\
\quad(\text { SU.VI.MAX) }\end{array}$ & France & Prospective (follow-up 13 years) & 3,083 & Cognitive performance & No \\
\hline
\end{tabular}

WHICAP Washington Heights-Inwood Columbia Aging Project, AD Alzheimer's disease, HPF Health Professionals Follow-up Study, NHS Nurses' Health Study, MCI mild cognitive impairment, CHAP Chicago Health and Aging Project, SU.VI.MAX supplementation with vitamins and mineral antioxidants 
in patients suffering from Alzheimer's disease again in the WHICAP cohort [19]. A total of 192 patients with a diagnosis of Alzheimer's disease were followed every 1.5 years for 4.4 years. At the end of the follow-up, 85 (44 \%) patients died. By analyzing the adherence of patients to Mediterranean diet, it became evident that those in the highest tertile of adherence at baseline had a significantly lower mortality risk (HR 0.27; $95 \%$ CI 0.1-0.69) with a 4-year longer survival. The present analysis suggested, for the first time, a possible relationship between adherence to the Mediterranean diet not only with the risk of occurrence but also with mortality from Alzheimer's disease, thus extending the finding to a secondary prevention level.

In addition, the same authors published another study showing that adherence to the Mediterranean diet combined with physical activity was independently associated with a reduced risk of Alzheimer's disease in the same cohort [20]. During a mean follow-up of 5.4 years, patients both adhering to the Mediterranean diet and doing physical activity had a lower risk of Alzheimer's disease (HR 0.65; $95 \%$ CI 0.44-0.96) compared with individuals neither adhering to the diet nor doing physical activity.

That's not all; the beneficial effect of the Mediterranean diet in the WHICAP cohort was found not only on the incidence of Alzheimer's disease but also on cognitive decline, as measured by reduction in the score of a cognitive function test, such as the Mini-Mental State Examination test [21]. This study considered a cohort of 1,393 patients who had a normal level of cognitive function at baseline; their adherence to the Mediterranean diet was related to reduction in the risk of experiencing cognitive decline (HR 0.52 ; $95 \%$ CI $0.3-0.91$ ). Indeed, after 4.5 years of followup, 275 subjects developed mild cognitive impairment and those in the highest tertile of adherence score to Mediterranean diet were significantly protected versus such cognitive impairment. Moreover, an additional result of the present study was that, among the population of patients with mild cognitive impairment at baseline $(n=482)$, those who were in the highest tertile of adherence to Mediterranean diet had less risk of developing Alzheimer's disease (HR 0.52; $95 \%$ CI 0.3-0.91).

During the same year, another cohort study that analyzed the possible association between adherence to Mediterranean diet and cognitive decline was published [22]. This prospective cohort study was performed in 1,410 French subjects followed-up for 5 years. The authors analyzed subjects who were enrolled within the Three-City Cohort, a prospective study of vascular risk factors of dementia, between 2001 and 2002 and reexamined at least once over 5 years. After a median follow-up time of 4.1 years, the adherence to Mediterranean diet was inversely related to cognitive decline, thus demonstrating that a higher Mediterranean diet score was associated with a slower cognitive decline. However, by analyzing the possible association between adherence to Mediterranean diet and risk of incident dementia, no significant association was found (HR 1.12; $95 \%$ CI 0.6-2.1). This study, which is actually the only prospective study that examined the relationship between adherence to the Mediterranean diet and cognitive decline in Europe, partly confirms the results of previous studies conducted within the WHICAP study, even if no significant association with dementia has been demonstrated.

One year later, in 2010, a prospective study conducted in a population of 1,969 elderly subjects living in Minnesota was released [23]. It analyzed the possible association between adherence to the Mediterranean diet and mild cognitive impairment. Among 1,141 subjects who had mild cognitive impairment or were cognitively normal at baseline, 93 manifested mild cognitive impairment and 23 reported a diagnosis of dementia. After 2.2 years of follow-up, patients in the highest tertile of adherence to the Mediterranean diet showed a trend for reduced risk of occurring mild cognitive impairment or dementia (HR 0.75; $95 \%$ CI 0.46-1.21), which did not reach statistical significance. Similar negative results were reported in two other prospective studies. The first, conducted in Australia, analyzed 1,528 subjects who were evaluated for adherence to the Mediterranean diet in association to mild cognitive impairment at follow-up [24]. After 4 years of follow-up, adherence to the Mediterranean diet did not correlate with cognitive decline (OR 1.2; $95 \%$ CI 0.98 $1.47)$, although a trend for significance was reported $(p=0.08)$. The second study reported an analysis of the SU.VI.MAX Study, an intervention study of supplementation with vitamin and mineral antioxidants [25••]. In this study, the authors investigated the possible association between adherence to Mediterranean diet and cognitive performance detected using six neuropsychological tests, despite not strictly related to the primary aim of the study. After adjustment for potential confounders, no association between adherence to Mediterranean diet and a composite score of cognitive tests was reported.

On the contrary, different results were observed recently by a population study conducted in Chicago, where adults $>65$ years showed that adherence to a Mediterraneantype dietary pattern was able to reduce the rate of cognitive decline [26]. The study population included more than 4,000 subjects evaluated for an average period of approximately 8 years. After adjustment for all the possible confounders, the authors were able to report a significant association between a higher adherence score to the Mediterranean diet and a slower rate of cognitive decline.

In 2007, a prospective study of 49,692 men and 81,676 women attending the Health Professionals and Nurses' Health Study extended the possible beneficial effects of the Mediterranean diet to another neurodegenerative disease 
of great interest, such as Parkinson's disease [27]. After 16 years of follow-up, the authors found 508 new cases of Parkinson's disease and were able to show an inverse association between adherence to the Mediterranean diet and risk of this disease. In fact, subjects who were in the highest quintile of adherence to the Mediterranean diet score showed a $25 \%$ reduction in the risk of Parkinson's disease compared with those who had a lower score of adherence (relative risk (RR) 0.75 ; $95 \%$ CI 0.57-1).

With the goal of analyzing altogether the available published studies that reported the possible relationship between adherence to Mediterranean diet and neurodegenerative diseases, our group decided to perform two meta-analyses among the available prospective cohort studies. The overall analysis resulting from the meta-analysis was able to demonstrate the protective effect of adherence to the Mediterranean diet in relation to neurodegenerative diseases. The first analysis of 5 prospective studies including a total of 133,626 subjects showed that an increase of 2 points in the adherence to the Mediterranean diet resulted in a $13 \%$ reduction in the risk of suffering from these diseases (RR 0.87; $95 \%$ CI 0.8-0.96; $p<0.0001$ ) [28]. An update of the present meta-analysis has been performed with the most recent studies 2 years later. The updated analysis confirmed the previous results, with a similar association (RR 0.87; $95 \%$ CI 0.81-0.94; $p<0.0001$ ). As a result, a 2-point increase in the adherence to Mediterranean diet determines a $13 \%$-reduction in the risk of occurrence of neurodegenerative diseases [29].

\section{Conclusions}

The association between adherence to the Mediterranean diet and prevention of dementia, Alzheimer's disease, and mild cognitive impairment has been investigated in several studies, indicating that the issue is extremely interesting and appealing. However, findings are not conclusive and some conflicting evidence arose. Many of the published studies came from the same cohorts, located in non-Mediterranean regions; therefore, replications in other populations, likely of the Mediterranean basin, are extremely needed to validate these preliminary data. In addition, the variability in the neurological tests used to examine the mild cognitive impairment does not help physicians and researchers to uniform and standardize the results. On the other hand, the supposed beneficial effect of a Mediterranean diet model on such diseases is extremely relevant because of the high impact that such preventive approach might have in the whole population and needs to be better defined and studied. All possible efforts are warranted by investigators, physicians, and institutions to seek the real nature of the relationship between this model of diet and cognitive health.
Conflict of Interest Francesco Sofi declares that he has no conflict of interest.

Claudio Macchi declares that he has no conflict of interest.

Alessandro Casini declares that he has no conflict of interest.

\section{References}

Papers of particular interest, published recently, have been highlighted as:

- Of importance

•- Of outstanding importance

1. Ferri CP, Prince M, Brayne C, et al. Global prevalence of dementia: a Delphi consensus study. Lancet. 2005;366:2112-7.

2. Daviglus ML, Bell CC, Berrettini W, et al. Risk factors and preventive interventions for Alzheimer disease: state of the science. Archiv Neurol. 2011;68:1185-90.

3. Plassman BL, Williams Jr JW, Burke JR, et al. Systematic review: factors associated with risk for and possible prevention of cognitive decline in later life. Ann Intern Med. 2010;153:182-93.

4. Morris MC. Nutritional determinants of cognitive aging and dementia. Proc Nutr Soc. 2012;71:1-13.

5. Sofi F. The Mediterranean diet revisited: evidence of its effectiveness grows. Curr Opin Cardiol. 2009;24:442-6.

6. Sofi F, Macchi C, Abbate R, Gensini GF, Casini A. Effectiveness of the Mediterranean diet: can it help delay or prevent Alzheimer's disease? J Alzheimers Dis. 2010;20:1-7.

7. Solfrizzi V, Panza F, Frisardi V, Seripa D, Logroscino G, Imbimbo BP, et al. Diet and Alzheimer's disease risk factors or prevention: the current evidence. Expert Rev Neurother. 2011;11:677-708.

8. Petersen RC. Clinical practice. Mild cognitive impairment. N Engl J Med. 2011;364:2227-34.

9. Palmer K, Backman L, Winblad B, Fratiglioni L. Mild cognitive impairment in the general population: occurrence and progression to Alzheimer disease. Am J Geriatr Psychiatry. 2008;16:603-11.

10. • Feart C, Samieri C, Alles B, Barberger-Gateau P. Potential benefits of adherence to the Mediterranean diet on cognitive health. Proc Nutr Soc. 2013;72:140-52. A well-written review on the relationship between Mediterranean diet and cognitive disorders.

11. Logroscino G, Marder K, Cote L, Tang MX, Shea S, Mayeux R. Dietary lipids and antioxidants in Parkinson's disease. Ann Neurol. 1996;39:89-94.

12. The diet and all-cause death rate in the Seven Countries Study. Lancet 1981, 2: 58-61.

13. Trichopoulou A, Costacou T, Bamia C, Tricopoulou D. Adherence to a Mediterranean diet and survival in a Greek population. N Engl J Med. 2003;348:2599-608.

14. Panagiotakos DB, Pitsavos C, Arvaniti F, Stefanadis C. Adherence to the Mediterranean food pattern predicts the prevalence of hypertension, hypercholesterolemia, diabetes and obesity, among healthy adults; the accuracy of the MedDietScore. Prev Med. 2007;44:335-40.

15. Dominguez LJ, Bes-Rastrollo M, de la Fuente-Arrillaga C, Toledo E, Beunza JJ, Barbagallo M, et al. Similar prediction of decreased total mortality, diabetes incidence or cardiovascular events using relative- and absolute-component Mediterranean diet score: the SUN cohort. Nutr Metab Cardiovasc Dis. 2012;2488:2488. doi:10.1016/j.numecd.2011.10.009.

16. Fidanza F, Alberti A, Lanti M, Menotti A. Mediterranean adequacy index: correlation with 25 -year mortality from coronary heart disease in the seven countries study. Nutr Metab Cardiovasc Dis. 2004;14:254-8. 
17. Scarmeas N, Stern Y, Tang MX, Mayeux R, Luchsinger JA. Mediterranean diet and risk for Alzheimer's disease. Ann Neurol. 2006;59:912-21.

18. Scarmeas N, Stern Y, Mayeux R, Luchsinger JA. Mediterranean diet, Alzheimer disease, and vascular mediation. Arch Neurol. 2006;63:1709-17.

19. Scarmeas N, Luchsinger JA, Mayeux R, Stern Y. Mediterranean diet and Alzheimer disease mortality. Neurology. 2007;69:1084-93.

20. Scarmeas N, Luchsinger JA, Schupf N, et al. Physical activity, diet, and risk of Alzheimer disease. JAMA. 2009;302:627-37.

21. Scarmeas N, Stern Y, Mayeux R, et al. Mediterranean diet and cognitive impairment. Arch Neurol. 2009;66:216-25.

22. Feart C, Samieri C, Rondeau V, et al. Adherence to a Mediterranean diet, cognitive decline, and risk of dementia. JAMA. 2009;302: 638-48.

23. Roberts RO, Geda YE, Cerhan JR, et al. Vegetables, unsaturated fats, moderate alcohol intake, and mild cognitive impairment. Dement Geriatr Cogn Disord. 2010;29:413-23.
24. Cherbuin N, Amstey KJ. The Mediterranean diet is not related to cognitive change in a large prospective investigation: the PATH through life study. Am J Geriatr Psychiatry. 2012;20:635-9.

25. • Kesse-Guyot E, Andreeva VA, Lassale C, et al. Mediterranean diet and cognitive function: a French study. Am J Clin Nutr. 2013;97:369-76. One of the only two available studies conducted in an European population.

26. Tangney CC, Kwasny MJ, Li H, et al. Adherence to a Mediterranean-type dietary pattern and cognitive decline in a community population. Am J Clin Nutr. 2011;93:601-7.

27. Gao X, Chen H, Fung TT, et al. Prospective study of dietary pattern and risk of Parkinson disease. Am J Clin Nutr. 2007;86:1486-94.

28. Sofi F, Cesari F, Abbate R, et al. Adherence to Mediterranean diet and health status: a meta-analysis. Br Med J. 2008;337:a1344.

29. Sofi F, Abbate R, Gensini GF, Casini A. Accruing evidence about benefits of adherence to the Mediterranean diet on health: an updated systematic review and meta-analysis. Am J Clin Nutr. 2010;92:1189-96. 